\title{
Experience of Photopneumatic Therapy in Taiwanese Acne Patients
}

\author{
Haw-Yueh Thong1,2, En Jen ${ }^{3}$, Chieh Jen ${ }^{4}$, Chieh-Chen Huang2* \\ ${ }^{1}$ Dr. Kelly Tang's Dermatology Clinic, Taipei, Taiwan \\ ${ }^{2}$ Department of Dermatology, Shin-Kong Wu Ho-Su Memorial Hospital, Taipei, Taiwan \\ ${ }^{3}$ Institute of Biomedical Electronics and Bioinformatics, National Taiwan University, Taipei, Taiwan \\ ${ }^{4}$ Graduate Institute of Hakka Social and Cultural Studies National Central University, Chung-Li, Taoyuan, Taiwan \\ Email: ${ }^{\text {drkellytang@gmail.com }}$
}

Received 10 October 2014; revised 12 November 2014; accepted 24 November 2014

Academic editor: Paul J. Higgins, Albany Medical College, USA

Copyright (C) 2014 by authors and Scientific Research Publishing Inc.

This work is licensed under the Creative Commons Attribution International License (CC BY).

http://creativecommons.org/licenses/by/4.0/

(c) (i) Open Access

\begin{abstract}
Acne is a disease of the pilosebaceous unit affecting both teenagers and adults. The treatment of acne is sometimes frustrating due to the emergence of antibiotic resistance, skin irritation and lack of novel therapy. Light and vacuum devices, also known as photopneumatic therapy, have been shown promising as a generally well-tolerated adjunctive treatment of acne vulgaris. We conducted a clinical study using such device to assess the efficacy and safety as an adjunctive treatment of acne in Taiwanese patients. Twenty patients received six sequential treatments every one to two weeks in the facial region. A $4 \times 4 \mathrm{~cm}^{2}$ area on cheek was chosen as a control area and was not treated with photopneumatic device. Safety parameters and adverse events were recorded. VISIA Complexion Analysis System was used for image analysis on the improvement of four parameters, namely, pigmentation, pore reduction, redness and porphyrin levels. There was an overall improvement in pigmentation, pore size, redness and porphyrin levels on treatment areas. The treatment areas showed statistically significant improvement $(p<0.05)$ in pigmentation and pore sizes compared with the control areas using paired samples T-test. Mild and self-limiting localized adverse events, mostly focal petechiae or mild irritation, were reported. Our study has demonstrated that photopneumatic therapy is an effective, safe and relatively well-tolerated procedure in Taiwanese acne patients.
\end{abstract}

\section{Keywords}

Taiwanese, Ethnic Skin, Acne Vulgaris, Propionibacterium acnes, Drug Resistance, Vacuum and Light Device, Photopneumatic Therapy

\footnotetext{
${ }^{*}$ Corresponding author.
}

How to cite this paper: Thong, H.-Y., Jen, E., Jen, C. and Huang, C.-C. (2014) Experience of Photopneumatic Therapy in Taiwanese Acne Patients. Journal of Cosmetics, Dermatological Sciences and Applications, 4, 332-338. 


\section{Introduction}

Acne is a disease of the pilosebaceous unit affecting both teenagers and adults. It is one of the most common disorders treated by the medical professions as well as self-treatment using over the counter remedies. It can manifest itself as comedones, papules, pustules, nodules and deep cysts. There are numerous treatments available, which can vary from, but not limited to, systemic and topical antibiotics, topical benzoyl peroxide, topical retinoids and oral isotretinoin. Many of these treatments are effective if used for a sustained period of time. The common problems in the treatment of acne vulgaris include drug resistance, skin irritation, frequent relapse, slow onset of treatment response, etc. [1].

All acne lesions start with the formation of a microcomedone created by hyperproliferation of the follicular epithelium [2]. At this early stage, inflammatory processes and activation of the vascular endothelium occur, resulting from various reasons. Changes in the concentration of the lipids in sebum may be one of the factors [3]. The inflammatory cytokines residing in the sebum remain to further stimulate the sebocytes to produce sebum and also cause local tissue destruction.

Blue light devices, pulse light device with aminolevulinic acid (ALA) and intense pulsed lights devices (IPLs) are common devices used in treatment of acne vulgaris, by eliminating Propionibacterium acnes (pAcnes) [4][7]. But these devices experience common problems. For example, even if the light reaches the porphyrins and destroys the pAcnes, it cannot destroy all the pAcnes. The residual bacteria colonies immediately start repopulating the lesion. As long as there is sufficient sebum remaining, the conditions for the pAcnes population to rebound are ideal.

The Acleara Acne Clearing System (Palomar Medical Technologies, Burlington, MA) is a novel photopneumatic therapy system combining both filtered broad band light and vacuum [8]. The device delivers broad band light ranged $500-1200 \mathrm{~nm}$ and delivers a gentle vacuum suction on the treatment area. When the vacuum activates, it generates up to 3 psi pressure in a confined treatment area which effectively lifts the dermal structures towards the epidermis and expels follicular contents onto the skin's surface thereby clearing the pore. The effect of vacuum also stretches the skin $25 \%-35 \%$, reducing effective melanin concentration in the epidermis, which increases the transmission of energy to the targeted structures. This elevation of the pilosebaceous unit results in more light delivered to target structures more efficiently as less energy is lost in the process of transmission. Along with the targeted heating of the dermis, the endogenous effect of light activates porphyrins to destroy pAcnes bacteria and reduce sebum production. The pressure caused by the vacuum combined with the heat generated by the absorption of light removes the follicular contents. Sebaceous material can be observed as it is forced out onto the surface of the skin. In addition to the sebaceous contents, pAcnes are removed both mechanically and thermally from the active acne lesions. Studies on Asian populations and other ethnic skin are minimal [9] [10].

\section{Aim}

The aim of this work is to evaluate the efficacy and safety of photopneumatic therapy on Taiwanese acne patients.

\section{Methods}

\subsection{Subjects}

This prospective study was conducted in a dermatologic clinic in Taipei City, Taiwan. Healthy non-pregnant individuals with mild to moderate acne, who have received oral/topical antibiotics and/or topical retinoid acid treatment but with unsatisfactory results were included. Twenty Taiwanese subjects (nine females and eleven males) were enrolled and received six sequential treatments every one to two weeks as tolerated. Treatment area with tattoos, scars, dermatitis, or open wounds and patients with a history of major diseases, diabetes mellitus, HIV infection, connective tissue disease, and malignant disease were excluded. Those with recent (within 3 months) or aesthetic laser/chemical peeling treatment and concurrent use of medication that could disrupt coagulation (e.g., aspirin, NSAIDs, warfarin) were also excluded. The patients were instructed to maintain a healthy lifestyle with balanced diet and avoid sleep deprivation or any kind of aesthetic laser/chemical peeling treatment during treatment and follow-up. The patients were allowed to use topical clindamycin gel/ topical adapalene on a short-term basis ( 3 - 5 days) and on focal areas should they experience severe flare-ups of the inflammato- 
ry/comedonal conditions. All patients provided written informed consent.

\subsection{Preparation and Treatment}

Before treatment, a thorough evaluation of the patient's medical history was conducted. A $4 \times 4 \mathrm{~cm}^{2}$ area on cheek was chosen as a control area which will not be treated during the trial period. Clinical examination of the treatment areas and control areas were recorded, followed by photographic imaging, and image analysis with VISIA Complexion Analysis System (Canfield Scientific Inc., New Jersey).

\subsection{Treatment Protocol}

The treatment area was first cleansed with a mild cleanser prior to treatment. All patients were photographed using the VISIA Complexion Analysis System. While the manufacturer's treatment protocol suggests steaming as a way to soften sebum and facilitate the extraction of comedones, we skipped the step due to time constraint in our daily practice. Energy settings were selected based on skin types. Vacuum settings were set to low (S1-2) on delicate areas such as the forehead and temples. All patients were treated using a double pulse with two passes over the entire face sparing the $4 \times 4 \mathrm{~cm}^{2}$ control area. Patients were treated at every one-to-two weeks for six treatments based on the severity of acne and level of tolerability. No external cool sprays, gels or anesthetics were used or required.

\section{Assessments and Statistical Analysis}

All of the patients underwent 6 treatments in the facial region, at one-to-two-week interval. No other aesthetic laser treatment was used during the study. Photographic documentation of the treated area was recorded prior to each treatment.

Efficacy was determined by photographic evaluation and measurements using VISIA Complexion Analysis System, while safety was determined by clinical findings and patients' report of any discomfort. Adverse events, including evaluation of patient's response and local skin reaction in the treatment area, were recorded throughout the study.

Statistical data analysis was performed using the SPSS software. Paired samples T-test were used to examine the data. All tests were two-tailed and $\mathrm{p} \leq 0.05$ was considered statistically significant. Due to the small sample size and non-normal data distribution, nonparametric tests were used to determine the difference between baseline and the last treatment. The Wilcoxon signed-rank test was used to compare two paired variables (pre-treatment and post-treatment measurements). Spearman's rho correlation coefficient was applied to test the strength of relationships among study parameters, while Friedman test was used to compare treatment shots among the six sessions.

\section{Results}

Twenty subjects completed the treatments. All subjects were Taiwanese, composed of eleven males and nine females, with a mean age of 23 years (male subjects, range 19 - 35 years old) and mean age of 26 years (female subjects, range 22 - 31 years old). All patients were of Fitzpatrick skin type III and all patients had moderate acne. Mean energy setting is at an energy setting of $6 \mathrm{~J} / \mathrm{cm}^{2}$, vacuum at level 2, and double pulse at $750 \mathrm{~ms}$.

The majority of patients noted a reduction in the number and severity of lesions, as represented in Figure 1. Treated patients also showed an improvement in the overall photographic presentations and quantitative parameters namely pigmentation, pore size, redness and reduction in porphyrin levels as captured by VISIA Complexion Analysis System. The majority of treated patients also reported a reduction in oiliness of the skin and an improvement in overall skin texture. Treatment providers noted a very dramatic reduction in blackheads and whiteheads that they had previously been unable to treat. Most patients were satisfied with treatment outcomes.

Side effects were transient and limited to the early part of our experience. The most common side effect was mild bruising which typically resolved within 48 - 72 hours post treatment. Once vacuum settings were adjusted and sensitive settings were used on areas such as the temples and forehead, no bruising occurred. Erythema typically resolved within 1 day of treatment.

Quantitative measurements using VISIA Complexion Analysis System showed at the treatment areas an improvement in pigmentation (overall improvement of $9 \%$ ), pore size $(12 \%)$, redness $(3 \%)$, and reduction in por- 


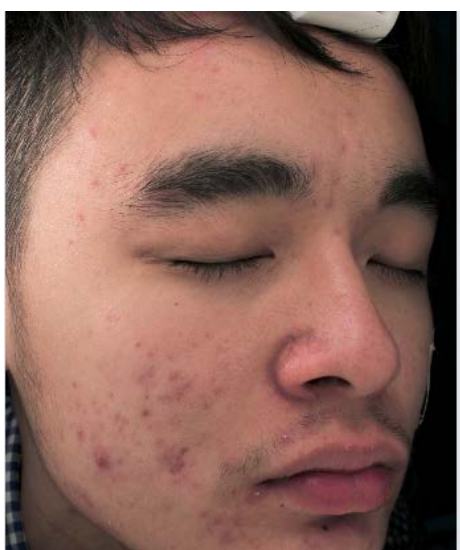

(a)

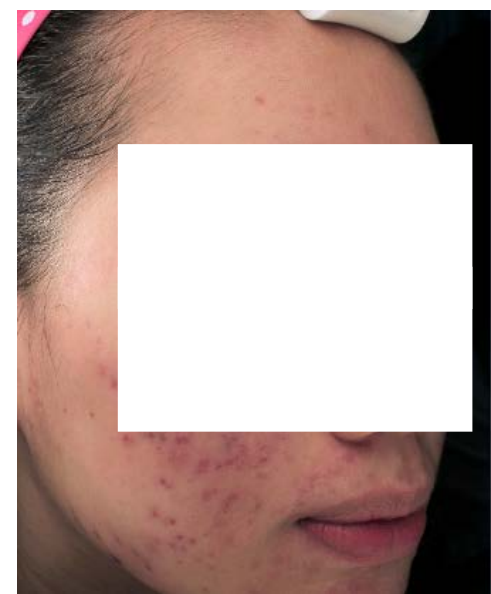

(c)

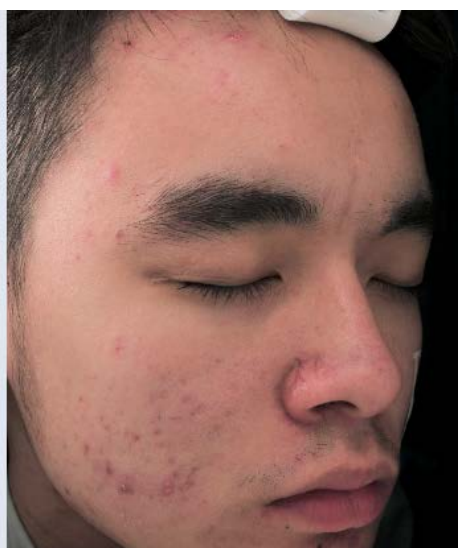

(b)

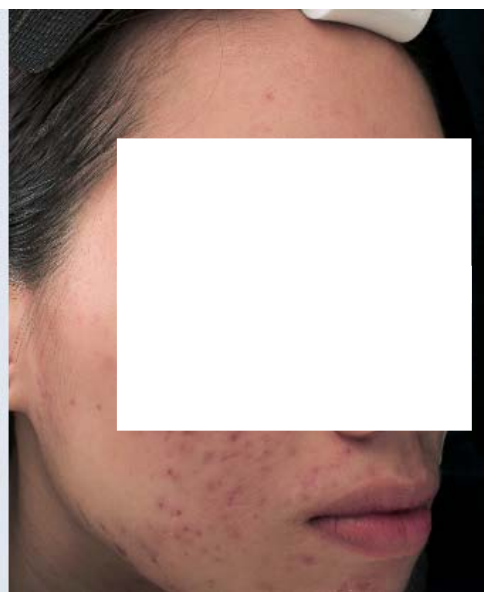

(d)

Figure 1. Photographic improvement captured by VISIA Complexion Analysis of a 18-yearold male subject ((a): before/baseline; (b): after 6 treatments spacing $1-2$ weeks apart) and a 22-year-old female subject ((c): before/baseline; (d): after 6 treatments) with moderate acne. An overall reduction on the amount of inflammatory lesions and comedonal lesions were noticeable at baseline ((a), (c)) and after the completion of 6 treatments ((b), (d)).

phyrin levels (17\%) as shown in Figure 2 and Figure 3. Due to the relatively small sample sizes, all data were pooled together for analysis regardless of the gender of the subjects. Statistical analysis using paired samples T-test showed significant improvement in pigmentation $(\mathrm{p}<0.05)$ and pore size $(\mathrm{p}<0.05)($ Table 1$)$. Whereas for the control areas there were no improvement in the four chosen parameters and there was no statistical significant improvement in all the parameters in the control areas (Table 2).

\section{Discussion}

Although the cause of the acne lesion is multifactorial, one major factor is an increase in sebum in the infundibulum unit. Removing the sebum/follicular contents is a sound method for reducing acne. By removing sebum/follicular contents, the level of pAcnes population is immediately reduced. The various inflammatory cytokines contained in the sebum are extracted. The inflammatory free fatty acids and peroxisomes are removed.

How the sebum is removed is also important. Since the dermal/epidermal barrier is compromised, any method that applies pressure on the sebum without a corresponding pressure on the dermis will force sebum out of the infundibulum into the dermis. This can occur using the traditional extraction methods. The key is to apply pressure on the dermis, thereby squeezing the sebum out of the infundibulum on to the surface of the skin. A vacuum chamber placed over the acne lesion would apply the right kind of pressure to the dermis surrounding the lesion and to the sebum contained in the lesion. 


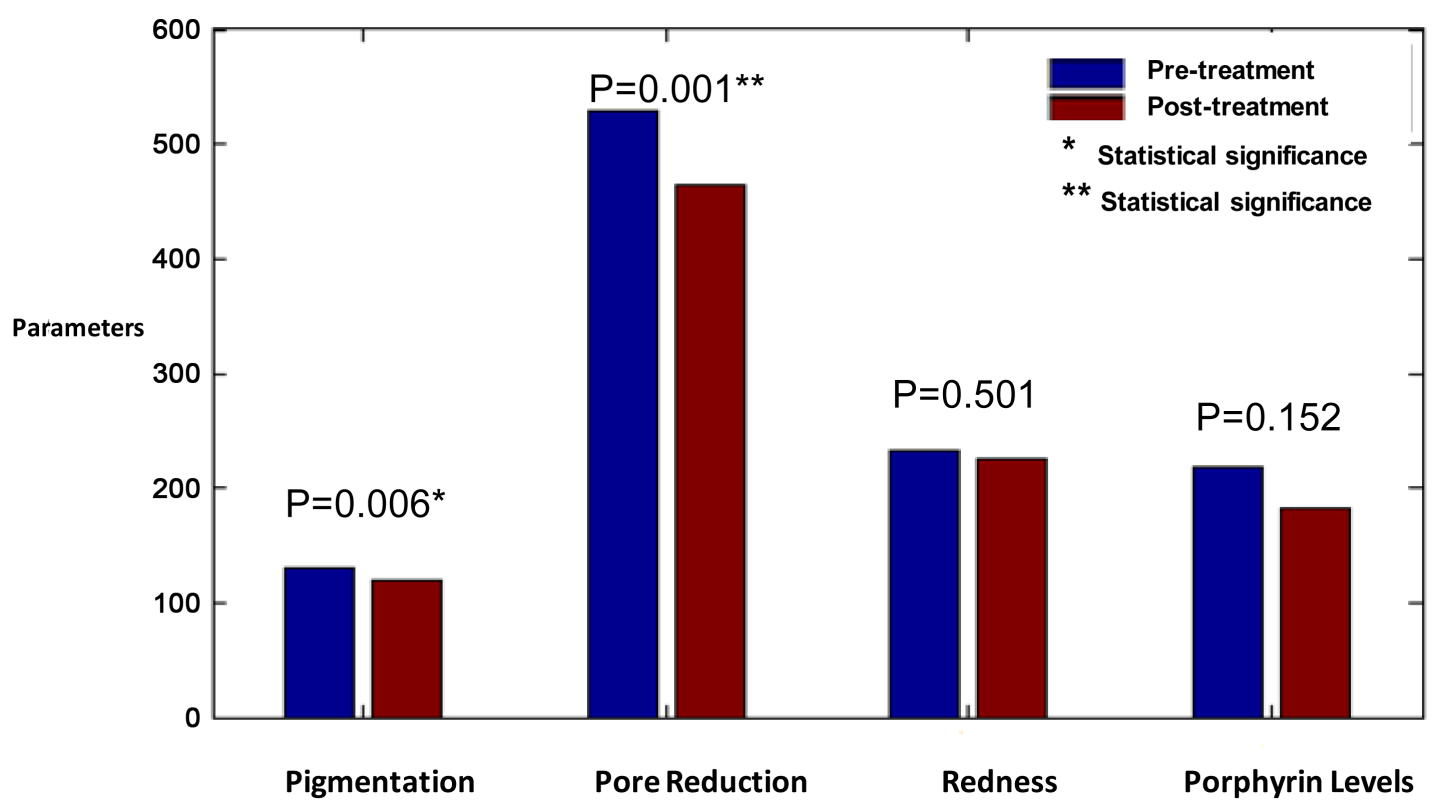

Figure 2. Before and after comparison of the improvement in parameters recorded by VISIA complexion analysis system $(n=20$, paired samples T-test was used for statistical analysis as shown in Table 1).

Table 1. Statistical analysis using paired samples - test on the parameters on treatment area (before $=$ baseline vs. after $=$ post 6 th treatments). Statistically significant improvement was noted in pigmentation $(p<0.05)$ and pore size $(p<0.05)$.

\begin{tabular}{|c|c|c|c|c|c|c|c|c|}
\hline \multicolumn{9}{|c|}{ Paired Samples T-Test of Treatment Area (Before vs. After) } \\
\hline & \multicolumn{5}{|c|}{ Variables } & \multirow{3}{*}{$\mathrm{T}$} & \multirow{3}{*}{$\begin{array}{l}\text { Degree } \\
\text { of Freedom }\end{array}$} & \multirow{3}{*}{$\begin{array}{r}\text { Significance } \\
\text { (Two-Tailed) }\end{array}$} \\
\hline & \multirow{2}{*}{ Average } & \multirow{2}{*}{$\begin{array}{c}\text { Standard } \\
\text { Deviation }\end{array}$} & \multirow{2}{*}{$\begin{array}{l}\text { Standard } \\
\text { Error }\end{array}$} & \multicolumn{2}{|c|}{$95 \%$ Confidence Interval } & & & \\
\hline & & & & Lower Level & Upper Level & & & \\
\hline Pigmentation (before-after) & 11.750 & 16.843 & 3.766 & 3.867 & 19.633 & 3.120 & 19 & 0.006 \\
\hline Pore reduction (before-after) & 61.600 & 71.095 & 15.897 & 28.327 & 94.873 & 3.875 & 19 & 0.001 \\
\hline Redness (before-after) & 6.700 & 43.701 & 9.772 & -13.753 & 27.153 & 0.686 & 19 & 0.501 \\
\hline Porphyrin level (before-after) & 37.450 & 112.273 & 25.105 & -15.095 & 89.995 & 1.492 & 19 & 0.152 \\
\hline
\end{tabular}

Table 2. Statistical analysis using paired samples T-test on the parameters on control area (before $=$ baseline vs. after $=$ post 6th treatments). No statistically significant improvement was noted in all the parameters in the control areas.

\begin{tabular}{|c|c|c|c|c|c|c|c|c|}
\hline \multicolumn{9}{|c|}{ Paired Samples T-Test of Control Area (Before vs. After) } \\
\hline & \multicolumn{5}{|c|}{ Variables } & \multirow{3}{*}{$\mathrm{T}$} & \multirow{3}{*}{$\begin{array}{c}\text { Degree } \\
\text { of Freedom }\end{array}$} & \multirow{3}{*}{$\begin{array}{l}\text { Significance } \\
\text { (Two-Tailed) }\end{array}$} \\
\hline & \multirow{2}{*}{ Average } & \multirow{2}{*}{$\begin{array}{l}\text { Standard } \\
\text { Deviation }\end{array}$} & \multirow{2}{*}{$\begin{array}{l}\text { Standard } \\
\text { Error }\end{array}$} & \multicolumn{2}{|c|}{$95 \%$ Confidence Interval } & & & \\
\hline & & & & Lower Level & Upper Level & & & \\
\hline Pigmentation (before-after) & 1.250 & 10.392 & 2.324 & -3.613 & 6.113 & 0.538 & 19 & 0.597 \\
\hline Pore reduction (before-after) & 4.900 & 66.587 & 14.889 & -26.264 & 36.064 & 0.329 & 19 & 0.746 \\
\hline Redness (before-after) & 20.600 & 58.008 & 12.971 & -6.548 & 47.748 & 1.588 & 19 & 0.129 \\
\hline Porphyrin level (before-after) & -11.400 & 61.376 & 13.724 & -40.125 & 17.325 & -0.831 & 19 & 0.416 \\
\hline
\end{tabular}




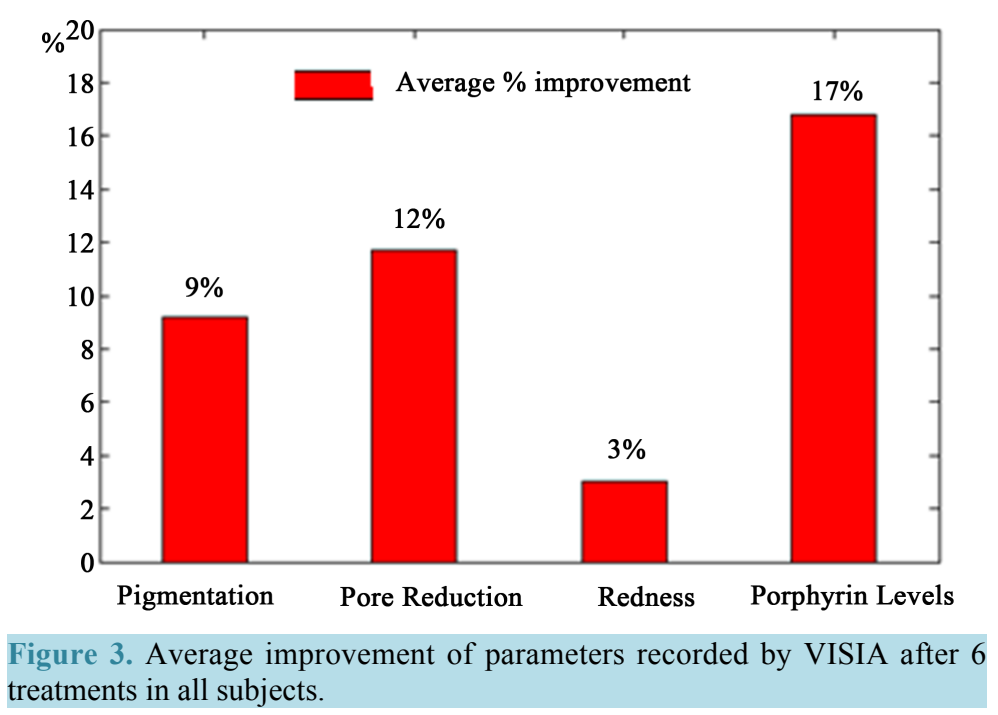

Although secondary to removing the sebum, the inflammation caused by acne has a major vascular component. Very small capillaries, produced in response to the inflammatory signaling, proliferate around and below the acne lesions. This increase in vasculature is detected as redness. Successful treatment to micro-comedones and comedones with the Acleara could not be done with previous devices, lessens the potential for an inflammatory and or cystic acne lesion to develop. The mechanism for treating these small comedones is postulated to be the removal of the sebum by the vacuum combined with the destruction of the small micro vasculature by the light. This micro vasculature is developing in response to the higher than normal concentration of various inflammatory factors as part of the cascading process that would ultimately lead to an inflammatory lesion.

The safety and efficacy of photopneumatic therapy for acne vulgaris has been studied. Adverse cutaneous events are few and range from erythema and blister to erosion and post-inflammatory hyperpigmentation. There is no downtime after acleara treatment, allowing patients to resume their treatment regimen and their normal activities immediately after because there is minimal skin texture change, irregularity, discoloration, ecchymosis, bruising, edema, and paresthesia. In the present study, there are only few cutaneous complications, which are mild and self-limiting. Most resolve spontaneously or with topical steroid treatment. Most are anticipated and told to patients before the procedure in this study.

For patients included in this study, videos, photos, and preliminary data on Taiwanese patients were used to explain the entire procedure, possible side effects, and results. The physician performed all of the procedures during treatment. Proper patient selection and communication can lessen over-expectation and are paramount to optimize treatment outcome and patient satisfaction. Multiple treatments may be necessary to obtain satisfactory results.

\section{Conclusion}

Studies of light-based acne treatments on Asian populations and other ethnic skin are minimal [9] [10]. Our study was the first to systematically analyze the efficacy of photopneumatic acne treatments in ethnic skin using VISIA Complexion Analysis System. We demonstrated that such treatments were well tolerated and effective for the treatment of a wide range of mild to severe acne. The most important, however, is the new ability to treat both open and closed comedones, something no other device has been able to do consistently. Given the positive outcome, we are investigating the use of the device as prophylactic on younger patients as a way to avert the development of cystic lesions. By treating the microcomedones before they can develop into inflammatory acne, we believe that photopneumatic acne treatments may either avert the manifestation of acne or at least reduce severity when it manifests. Such treatments most certainly will lessen the need for oral antibiotic therapy.

\section{Disclosures}

The authors have no conflict of interest to disclose.

There are no funding sources for this work. 


\section{References}

[1] Humphrey, S. (2012) Antibiotic Resistance in Acne Treatment. Skin Therapy Letter, 17, 9.

[2] Zouboulis, C.C., Eady, A., Philpott, M., Goldsmith, L.A., et al. (2005) What Is the Pathogenesis of Acne? Experimental Dermatology, 14, 145-152. http://dx.doi.org/10.1111/j.0906-6705.2005.0285a.x

[3] Kurokawa, I., Danby, F., Ju, Q., et al. (2009) New Developments in Our Understanding of Acne Pathogenesis and Treatment. Experimental Dermatology, 18, 821-832. http://dx.doi.org/10.1111/j.1600-0625.2009.00890.x

[4] Morton, C.A., Sholefield, R.D., Whitehurst, C., et al. (2005) An Open Study to Determine the Efficacy of Blud Light in the Treatment of Mild-to-Moderate Acne. Journal of Dermatological Treatment, 16, 219-223. http://dx.doi.org/10.1080/09546630500283664

[5] Elman, M. and Lebzelter, J. (2004) Light Therapy in the Treatment of Acne Vulgaris. Dermatologic Surgery, 30, 139146.

[6] Elman, M. and Lask, G. (2004) The Role of Pulsed Light and Heat Energy (LHE) in Acne Clearance. Journal of Cosmetic and Laser Therapy, 6, 91-95. http://dx.doi.org/10.1080/14764170410035584

[7] Goldman, M. and Boyce, S.M. (2003) A Single-Center Study of Aminolevulinic Acid and 417 nm Photodynamic Therapy in the Treatment of Moderate to Severe Acne Vulgaris. Journal of Drugs in Dermatology, 2, 393-396.

[8] Anderson, R., Kothavala, S. and Berger, R.S. (2013) An Advanced Vacuum and Light Technology for the Treatment of Acne Vulgaris.

[9] Dhawan, S. and Koreack, A. (2011) Tolerability in Asian Skin of a Consumer Blue Light Device for the Self-Treatment of Acne Vulgaris. Journal of the American Academy of Dermatology, 66.

[10] Davis, E.C. and Callender, V.D. (2010) A Review of Acne in Ethnic Skin. Pathogenesis, Clinical Manifestations, and Management Strategies. Journal of Clinical and Aesthetic Dermatology, 3, 4. 
Scientific Research Publishing (SCIRP) is one of the largest Open Access journal publishers. It is currently publishing more than 200 open access, online, peer-reviewed journals covering a wide range of academic disciplines. SCIRP serves the worldwide academic communities and contributes to the progress and application of science with its publication.

Other selected journals from SCIRP are listed as below. Submit your manuscript to us via either submit@scirp.org or Online Submission Portal.
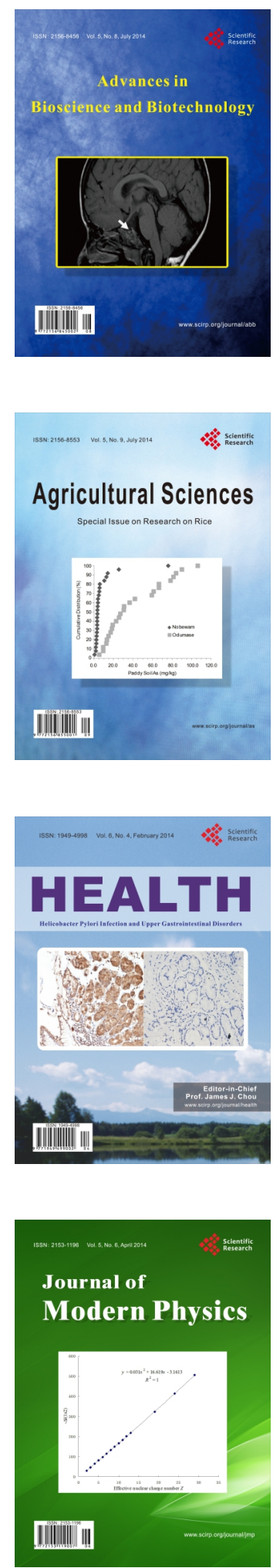
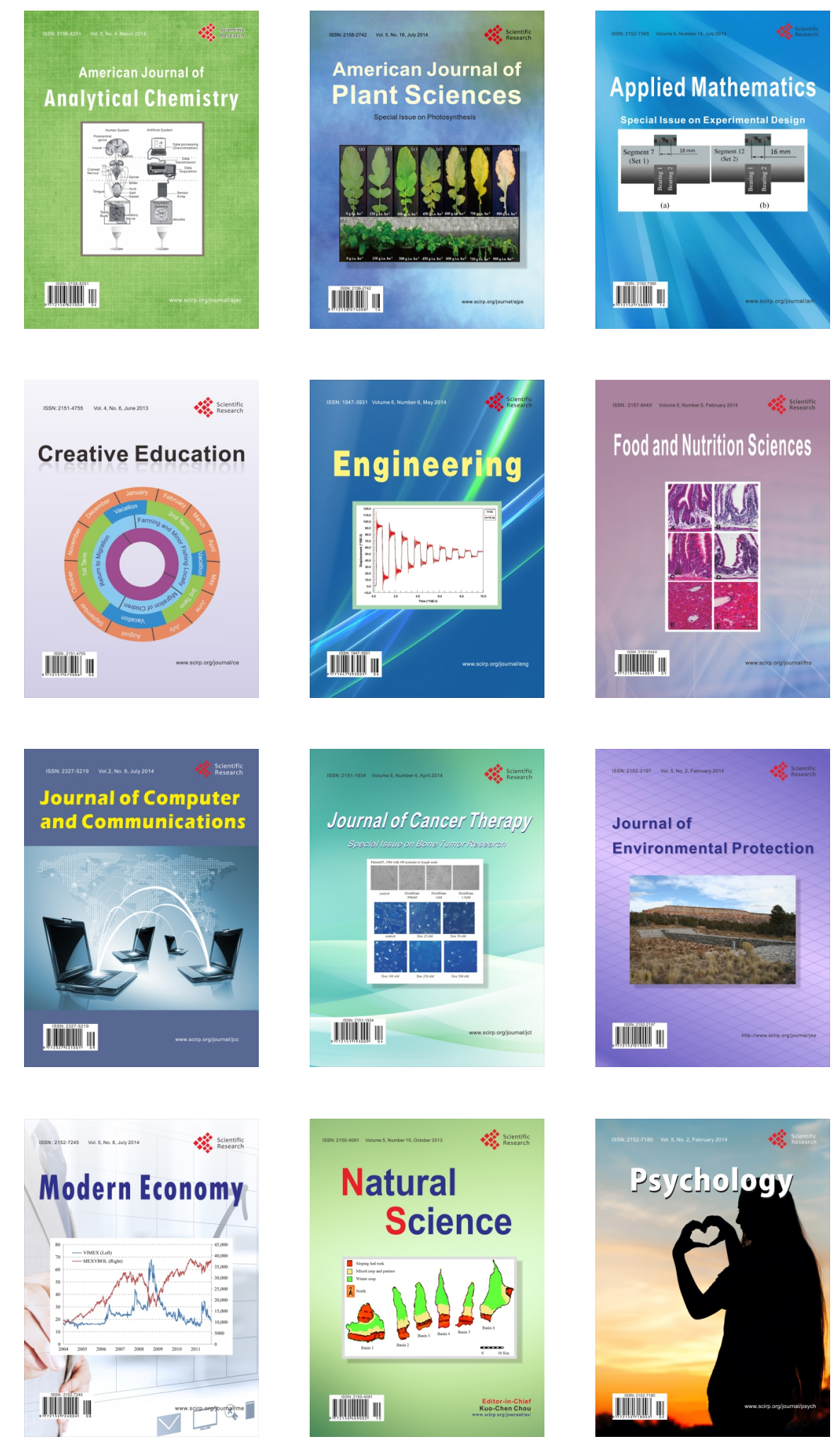